\title{
IMPLANTAÇÃO DE UM MÉTODO DE AVALIAÇÃO DIGITAL EM DISCIPLINAS DE FENÔMENOS FÍSICOS
}

\author{
GleyzerMartins-gmartins@ufu.br \\ UNIVERSIDADE Federal de Uberlândia- UFU \\ Rua 20, $n^{\circ} 1600$ - Bairro Tupã \\ CEP 38304-402- Ituiutaba - $M G$
}

Ana Luiza Gonçalves Cesar - Analu.gcs@gmail.com

UNIVERSIDADE Federal de Uberlândia- UFU

Rua 20, $n^{\circ} 1600$ - Bairro Tupã

CEP 38304-402- Ituiutaba - $M G$

Eugênio Pacceli Costa - eugenio@ufu.br

UNIVERSIDADE Federal de Uberlândia- UFU

Rua 20, $n^{\circ} 1600$ - Bairro Tupã

CEP 38304-402- Ituiutaba - MG

Resumo: O processo de ensino e aprendizado envolve necessariamente a utilização de um método de avaliação flexível e eficiente. Esse método deve possibilitar o diagnóstico do aprendizado e direcionar os alunos com desempenho não satisfatório para novos ciclos e aprendizado, avaliação e diagnóstico. As disciplinas de análise de fenômenos de transporte e operações unitárias apresentam turmas grandes com altas taxas de retenção e evasão. Neste trabalho, foi elaborado e implantado um método de avaliação, com a utilização de um software com propósito especifico, para a criação e correção dinâmica de atividades viabilizando os vários ciclos de aprendizado, avaliação e diagnóstico para turmas grandes. O software utilizado possibilitou a criação dinâmica de avaliações com base em um sorteio de questões de um banco de dados, realizando a correção automática e apresentando os resultados em uma interface de diagnóstico, permitindo, no final da avaliação, construir estratégias de aprendizado para direcionar os estudos dos discentes. Os resultados obtidos mostram que o método utilizado, por meio da interface amigável do software e com a apresentação organizada das questões e dos resultados da avaliação, teve uma boa aceitação por parte dos alunos.

Palavras-chave: Ciclos de Aprendizado. Avaliação e Diagnóstico. Avasão e Retenção escolar.

\section{INTRODUÇÃO}

O sistema de avaliação nos cursos de engenharia tem sido conduzido de forma soberana pelos docentes, buscando realizar a verificação do aprendizado com a classificação dos alunos em aprovados e reprovados. Esse processo de verificação somente classifica os alunos, sem a preocupação em diagnosticar os elementos falhos no aprendizado ou promover novos ciclos de aprendizado, avaliação e diagnóstico no mesmo semestre. A avaliação neste sentido cria um momento de conflito entre o docente, que idealiza um padrão de comportamento e de aprendizado, e os discentes, que buscam a aprovação a qualquer custo, lícita ou não, sem a preocupação com o aprendizado, Luckesi (2011). 
Luckesi (2010) aborda com clareza a importância de conduzir a avaliação para garantir o diagnóstico do aprendizado, oferecendo ao educando a oportunidade de melhorar a nota ou conceito. Nessa forma de avaliação existe a aproximação dos docentes e dos discentes para promover aprendizado do conteúdo, pois busca-se a cooperação entre as partes envolvidas para melhoria da qualidade do ensino e consequentemente a redução da retenção e evasão.

Outro aspecto relevante dos sistemas de avaliação, baseados em verificação, é que o aluno precisa participar da prova em condições físicas, mentais e psicológicas adversas. Outra particularidade desse sistema é que a recuperação da nota para aprovação eventualmente ocorre em outras avaliações dos conteúdos da disciplina, mascarando as falhas de aprendizado pelo emprego da média nas provas, mesmo com a classificação de aprovado do discente.

O método de avaliação com a utilização de um software, desenvolvido especificamente para esse fim, viabiliza a análise das falhas de aprendizado pelos docentes de forma rápida, efetiva e dinâmica mesmo para turmas grandes. Assim transformando a avaliação em um momento de construção do conhecimento, direcionando os discentes para novos ciclos e estratégias de aprendizado.

Em contraste, o uso de avaliações digitais não permitirá a análise do desenvolvimento dos conceitos no ato da avaliação, mesmo com a criação de questões em várias etapas ou itens. Entretanto é importante ressaltar que as avaliações digitais fazem parte de um sistema de avaliação diagnóstico que possibilita a realização de novos ciclos de aprendizado, avaliação e diagnóstico.

Com base nesse contexto, foi formulada a seguinte questão de pesquisa: o que pode ser feito para melhorar o desempenho dos alunos e diminuir a retenção em disciplinas de fenômenos físicos?

A partir dessa questão, foi estabelecido o seguinte objetivo de pesquisa: elaborar um método de avaliação dinâmico, claro e objetivo que permita ao aluno identificar seus pontos fracos e melhorar o seu desempenho na disciplina.

Para isso foram estabelecidos os seguintes objetivos específicos:

- Elaboração de um conjunto de diretrizes para o desenvolvimento do software necessário para implementação do método de avaliação digital;

- Criação de um software específico, com interface amigável, para aplicação de avaliações do aprendizado com respostas imediatas;

- Criação de formulários para apresentação organizada das questões e dos resultados das correções.

Neste sentido, esse trabalho consistiu em criar um método e desenvolver um software para a criação de avaliações digitais objetivando realizar o diagnóstico do aprendizado na disciplina de Operações Unitárias do curso de Engenharia de Produção da Universidade Federal de Uberlândia. O software permitiu a criação de avaliações com questões aleatórias baseadas em um banco de questões e a correção automática de questões numéricas com tolerância, além de apresentar os resultados das respostas dos alunos, das soluções do banco de dados e do status da correção: correto ou errado. As avaliações digitais buscam promover vários ciclos de aprendizado, avaliação e diagnóstico e a recuperação dos discentes evitando a retenção. $\mathrm{O}$ sistema de avaliação para a disciplina dividiu o conteúdo em três avaliações, em cada avaliação foi realizado em três etapas, possibilitando ao aluno realizar mais dois ciclos de aprendizado, avaliação e diagnóstico para cada conteúdo da disciplina. 


\section{FUNDAMENTOS TEÓRICOS}

\subsection{Avaliação}

Luckesi (2010) esclarece as diferenças entre a avaliação e a verificação do aprendizado, delimitando os aspectos da avaliação como um sistema de diagnóstico do ensino e a verificação como um sistema de classificação que estabelece a condição de aprovado ou de reprovado sem preocupação com aprendizado (JAVA,2020).

Lima (2016) afirma esse mesmo ponto de vista para a avaliação definindo a importância do diagnóstico do aprendizado para quantificar o conhecimento dos alunos, esclarecendo a diferença entre exame e avaliação diagnóstico.

No ato de avaliação, Luckesi (2000) recomenda a acolhida sem pré-julgamentos, o diagnóstico com a coleta de dados relevantes para o aprendizado e a elaboração de um instrumento para qualificar a aprendizagem fundamentado na teoria pedagógica para direcionar os objetivos da avaliação. Finalmente mostra a importância de se tomar uma decisão para completar o ato de avaliação, indicando os caminhos para o aprendizado.

Burini (2003) delimita o papel da avaliação no contexto do ensino como um mecanismo de retroalimentação do processo educacional, definindo os objetivos que as avaliações devem possuir para determinar as competências necessárias para a construção do conhecimento.

Aboutboul (1985) contextualiza o papel da avaliação da disciplina no processo de formação profissional, estabelecendo que a avaliação agrega valores no perfil profissional e destaca que a falta de consciência do docente deste processo pode comprometer os objetivos da disciplina.

De acordo com Pimenta, Anastasiou e Cavallet (2003), o ensino na Universidade constitui um processo de busca e construção científica e crítica ao conhecimento construído. Nesse sentido, apresentam várias características do ensino, entre as quais, destacam-se: a importância de criar e recriar situações de aprendizagem; valorizar a avaliação diagnóstica e compreensiva mais do que que a avaliação como controle; conhecer o universo de conhecimento dos alunos e desenvolver processos de ensino e aprendizagem interativos e participativos.

\subsection{Linguagem Java}

Java é uma linguagem de programação lançada pela Sun Microsystems em 1995, sendo uma linguagem rápida, segura e confiável. As aplicações variam de laptops a datacenters, consoles de games a supercomputadores científicos, telefones celulares à Internet (ORACLE, 2020).

Diferentemente de outras linguagens, o Java possui uma máquina virtual, Java Virtual Machine-JVM, que serve como um interpretador de código que pode ser aplicado em qualquer sistema operacional, (TECNOLOGIA E INFORMAÇÃ̃O, 2019), conferindo assim muita portabilidade à linguagem.

A linguagem Java, desta forma, apresenta uma sintaxe amigável, recursos orientados a objetos e a promessa de portabilidade para diferentes dispositivos, computadores, Internet e celulares, (SIERRA E BATES, 2005). Outra vantagem da linguagem é a vasta disponibilidade na internet de tutoriais, de códigos comentados e de bibliotecas para aplicação direta nas implementações de soluções para os programas desenvolvidos. 


\section{METODOLOGIA}

O desenvolvimento do software de avaliação digital deste trabalho está inserido dentro de um sistema de avaliação que busca o diagnóstico do aprendizado e que possibilite aos discentes realizar novos ciclos de aprendizado na disciplina de Operações Unitárias do curso de Engenharia de Produção.

Os ciclos de aprendizado, avaliação e diagnóstico foram aplicados para cada conteúdo das avaliações da disciplina em três momentos, possibilitando ao aluno mais duas oportunidades para o diagnóstico e a correção dos estudos. Os alunos puderam no semestre realizar nove ciclos de aprendizado, avaliação e diagnóstico.

A disciplina de Operações Unitárias está no oitavo período do projeto pedagógico do curso de Engenharia de Produção e no segundo semestre de 2019 foram matriculados 35 alunos com integralização curricular variada. Exigindo assim, para promover os ciclos de aprendizado, um sistema dinâmico de criação de avaliações, com correção automática e que permita ao docente no final da avaliação realizar o diagnóstico das falhas conceituais ou teóricas que os alunos apresentaram, direcionando os estudos destes no conteúdo da disciplina. Esse sistema dinâmico de criação, correção e diagnóstico da avaliação foi conseguido com o desenvolvimento do software de avaliação digital.

A implementação do software de avaliação digital pode trazer benefícios tanto pela facilidade de implementação da linguagem utilizada, quanto pela disponibilidade do uso em diferentes sistemas operacionais, além de poder disponibilizar para o usuário uma interface gráfica amigável com a apresentação organizada das questões e dos resultados das correções.

As diretrizes para o desenvolvimento do software foram:

- A criação de um banco de questões da disciplina, com os enunciados das questões, com os vários itens de acordo com os procedimentos de solução e com as respectivas soluções numéricas e unidades;

- A criação de uma avaliação com três questões do banco de questões selecionadas aleatoriamente buscando uma abordagem ampla do conteúdo com diferentes objetivos e complexidade;

- A implementação de uma interface gráfica que permita a coleta das informações dos alunos, a apresentação dos enunciados, das figuras explicativas e dos itens solicitados para cada questão, além dos campos para coleta das respostas dos alunos;

- A implementação de uma lógica de correção baseada na comparação das respostas com a solução do banco de questões, ressaltando que as correções das questões numéricas devem ser realizadas com tolerâncias definidas no banco de questões, evitando assim uma grande penalização na correção. Após a correção deve ser definido o status do item, correto ou errado, e também contabilizar a nota percentual da questão;

- A implementação de uma lógica para contabilizar a correção dos sistemas de unidades;

- A apresentação de uma aba com os resultados das correções das questões, contendo os itens, as respostas, as soluções e o status da correção, além da nota percentual da questão. Permitindo ao professor e ao aluno uma visão geral dos acertos e dos erros cometidos pelo aluno e ainda possibilitar ao professor identificar quais foram os itens desenvolvidos, a dimensão da discrepância numérica dos erros e as questões em branco;

- A criação de um mecanismo de back-up para recuperar as questões inicialmente selecionadas, caso ocorram panes no computador ou no software, evitando que o aluno faça novamente outras questões. 


\section{RESULTADOS}

Os resultados do trabalho mostram inicialmente as características da implementação do método com uso do software e em seguida são apresentadas a avaliação dos discentes a respeito da realização das avaliações digitais.

A primeira etapa de desenvolvimento do método foi a criação do banco de questões com os enunciados, os itens e as respostas. Foi definido para cada questão um banco de dados particular, com níveis de dificuldades distintos, evitando o desbalanceamento das avaliações entre os alunos. A Figura 1 apresenta o exemplo do banco de questões criado em arquivos texto.

Figura 1 - Exemplo do banco de dados de questões

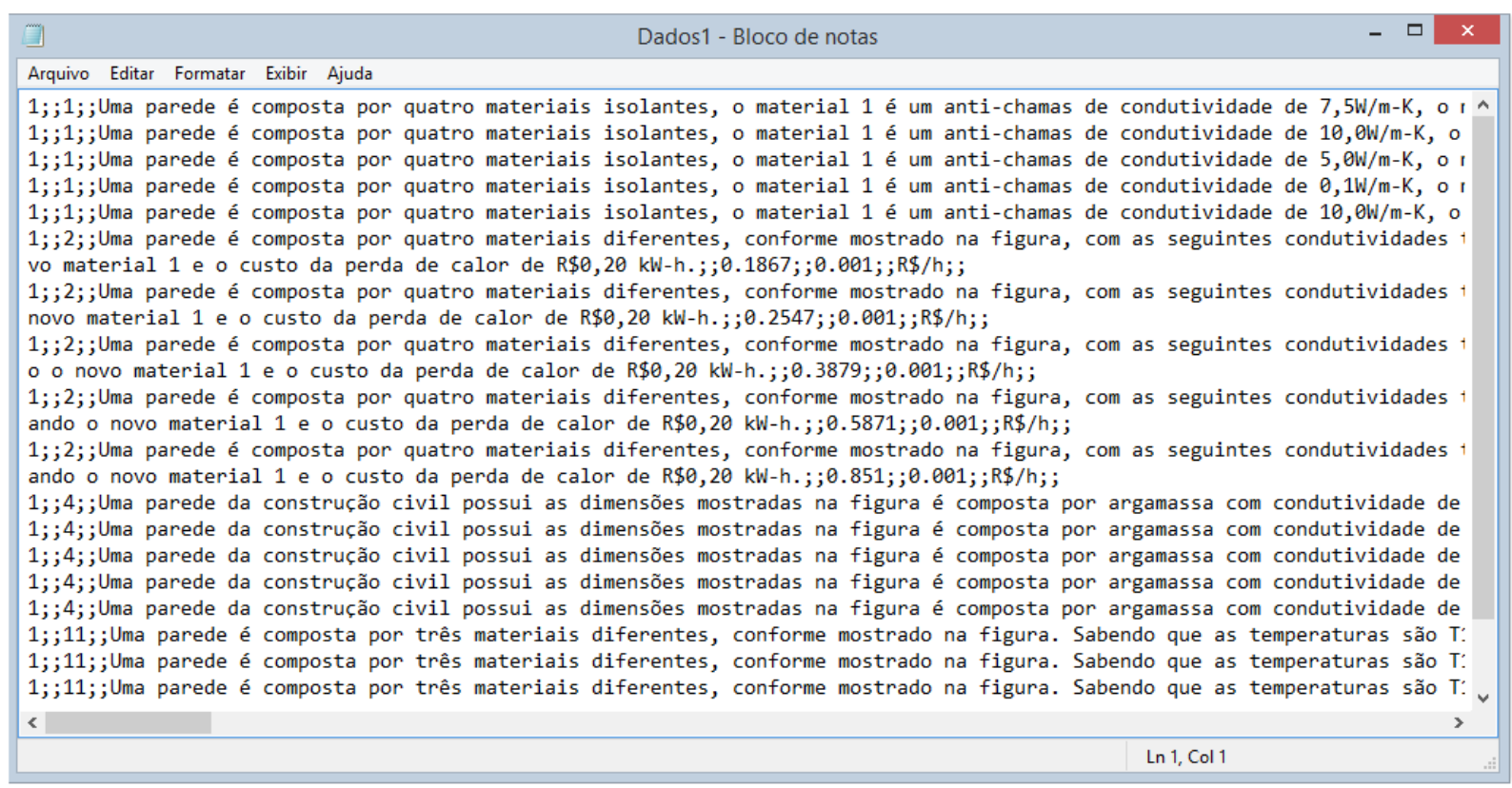

Fonte: autoria própria

A Figura 1 mostra que foi empregado dois caracteres “.,;” para separar os enunciados, os itens, as respostas e as unidades. Também foi desenvolvido um banco de figuras explicativas para cada questão.

Para o desenvolvimento da interface de coletas das informações dos alunos, apresentação das questões e dos resultados para o diagnóstico, foi empregado a divisão do software em abas, conforme pode ser visto nas Figuras: 2,3 e 4. 
Figura 2 - Interface gráfica

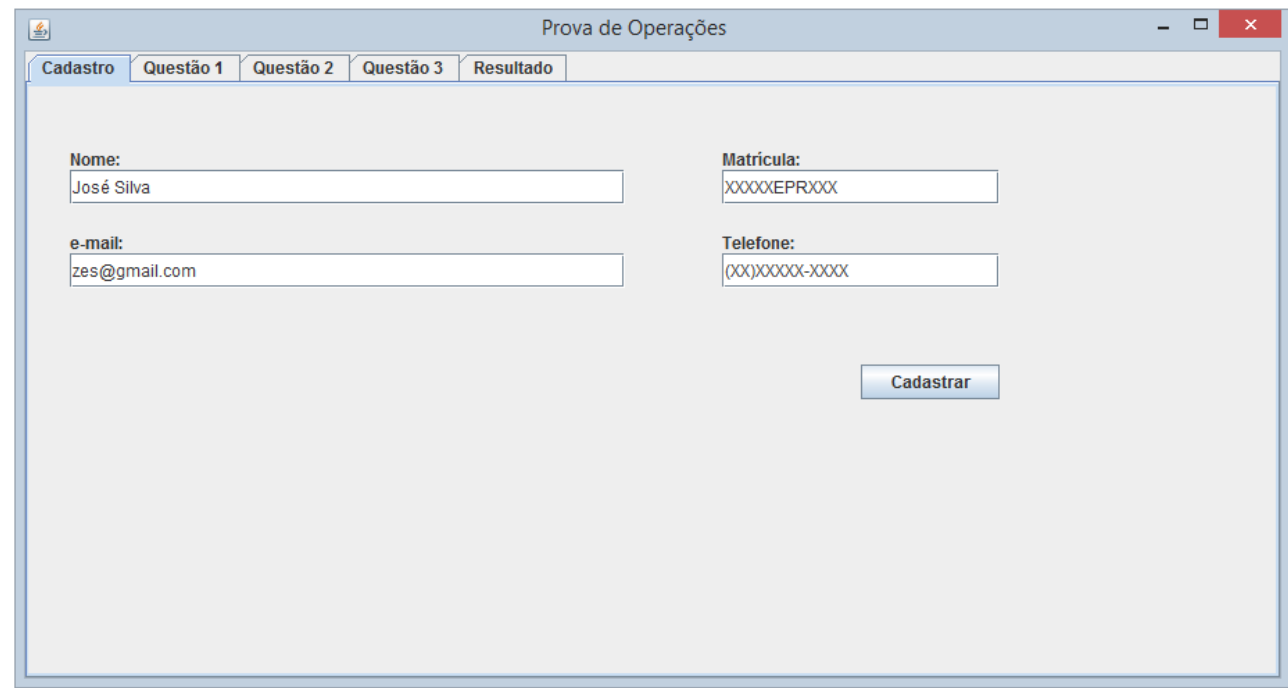

Fonte: autoria própria

A Figura 2 mostra a interface criada para a coleta das informações dos alunos, os campos coletam as informações e somente após o clicar em "Cadastrar" é habilitado o sistema de criação das questões.

Figura 3 - Questão 1

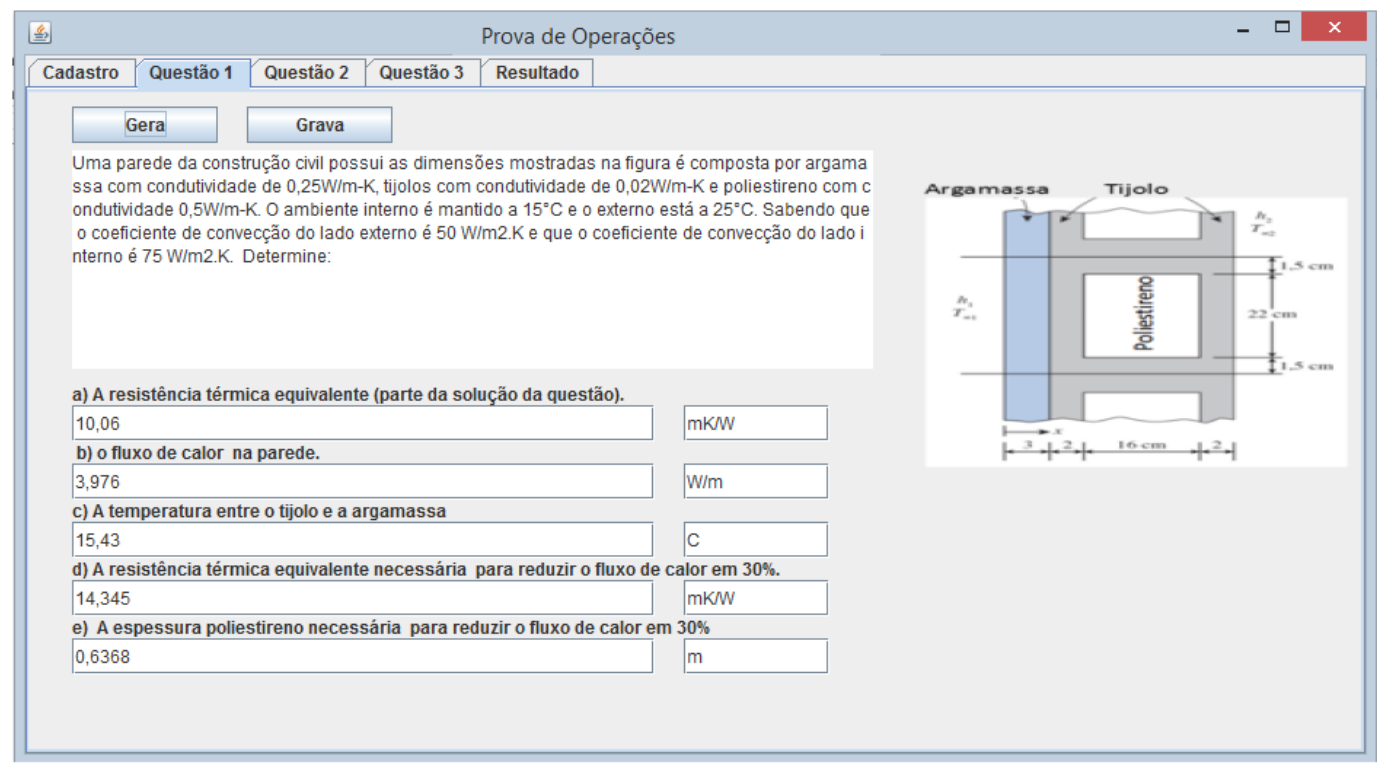

Fonte: autoria própria

A Figura 3 apresenta uma das interfaces de criação das questões, baseadas na seleção aleatória do banco de questões em que o aluno gera ao confirmar o cadastro. Ao final, são gravadas as suas respostas dos itens preenchidos. Observa-se que a interface disponibiliza um campo para o enunciado, para a figura explicativa, para os itens e para coleta das respostas. Neste ponto destaca-se a versatilidade da escolha da linguagem Java, pois ela permitiu a criação dinâmica de vários itens e campos de respostas dependentes da característica da questão. Como observado a questão 1 tem itens até a letra "e". Outras questões podem possuir itens até a letra "f".. 
Na Figura 4 tem-se a interface de apresentação dos resultados para o diagnóstico em que são mostrados na forma de tabelas os itens das questões com as respostas dos alunos, com as soluções do banco de questão e com o status da resposta.

Figura 4 - Resultados

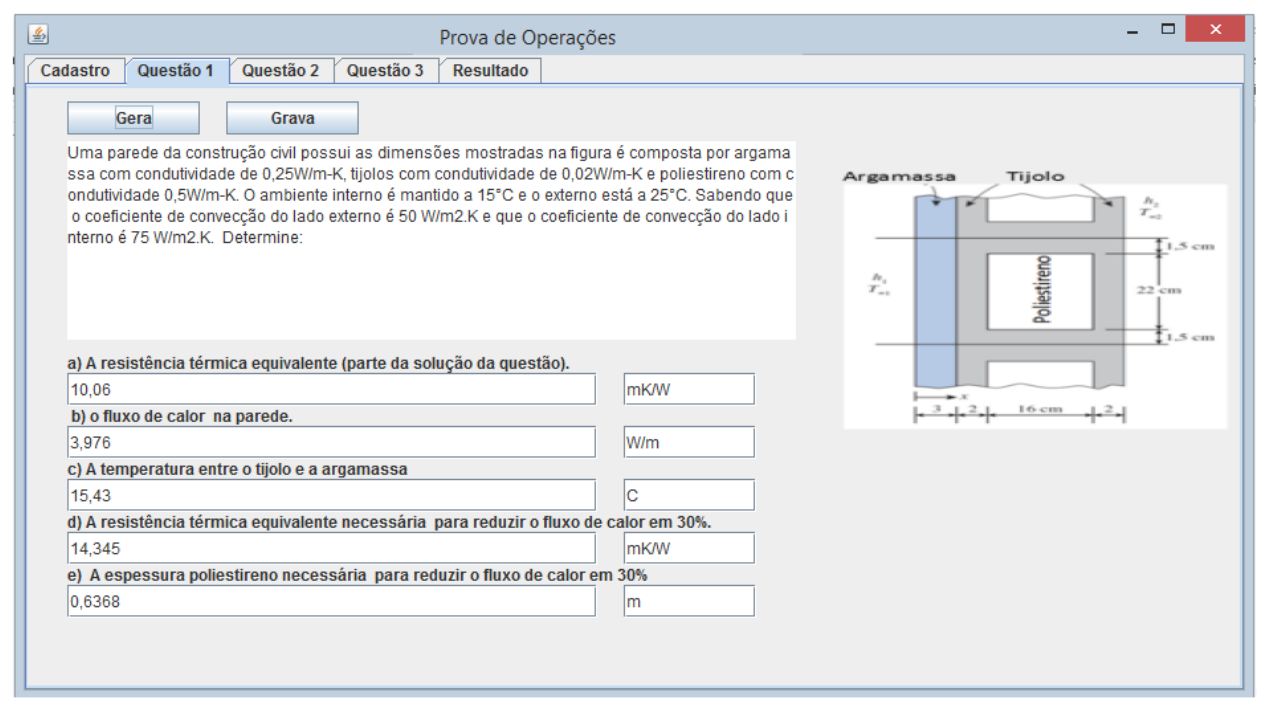

Fonte: autoria própria

A correção é realizada tanto para os valores numéricos quanto para o sistema de unidades e ao final o software permite a impressão da avaliação com as respectivas correções. A Figura 5 mostra um recorte de uma página do relatório gerado e disponível para impressão. As demais questões da avaliação são mostradas no mesmo formato da questão 01, dada pela Figura 5.

Figura 5 - Exemplo de impressão dos resultados

\section{Universidade Federal de Uberlândia Curso de Graduação em Engenharia de Produção Prova de Operações Unitárias}

Professor: Gleyzer Martins

Nota :49,44

\begin{tabular}{|l|l|l|l|}
\hline Nome: & José da Silva & Matricula: & XXXXXEPRXXX \\
\hline E-mail: & zes@gmail.com & Telefone: & $(X X) X X X X X-X X X X$ \\
\hline
\end{tabular}

Nota : 33,33

Questão 01 :Uma parede da construção civil possui as dimensões mostradas na figura é composta por argamassa com condutividade de $0,25 \mathrm{~W} / \mathrm{m}-\mathrm{K}$, tijolos com condutividade de $0,02 \mathrm{~W} / \mathrm{m}-\mathrm{K}$ e poliestireno com condutividade $0,5 \mathrm{~W} / \mathrm{m}-\mathrm{K}$. O ambiente interno é mantido a $15^{\circ} \mathrm{C}$ e o externo está a $25^{\circ} \mathrm{C}$. Sabendo que o coeficiente de convecção do lado externo é $50 \mathrm{~W} / \mathrm{m} 2 . \mathrm{K}$ e que o coeficiente de convecção do lado interno é $75 \mathrm{~W} / \mathrm{m} 2 . \mathrm{K}$. Determine:

a) A resistência térmica equivalente (parte da solução da questão).

Resposta:10.06[mK/W] Gabarito:10.06[mK/W] Correção:Correto

b) o fluxo de calor na parede.

Resposta:3.977[W/m] Gabarito:3.976[W/m] Correção:Correto

c) A temperatura entre o tijolo e a argamassa

Resposta:15.51[C] Gabarito:15.53[C] Correção:Correto

d) A resistência térmica equivalente necessária para reduzir o fluxo de calor em $30 \%$.

Resposta:14.35[mK/W] Gabarito:14.35[mK/W] Correção:Correto

e) A espessura poliestireno necessária para reduzir o fluxo de calor em $30 \%$

Resposta:0.6368[m] Gabarito:0.6368[m] Correção:Correto

Fonte: autoria própria 
No que tange a implementação da lógica de correção o software foi desenvolvido conforme o fluxograma da Figura 6.

Figura 6 - Lógica de correção

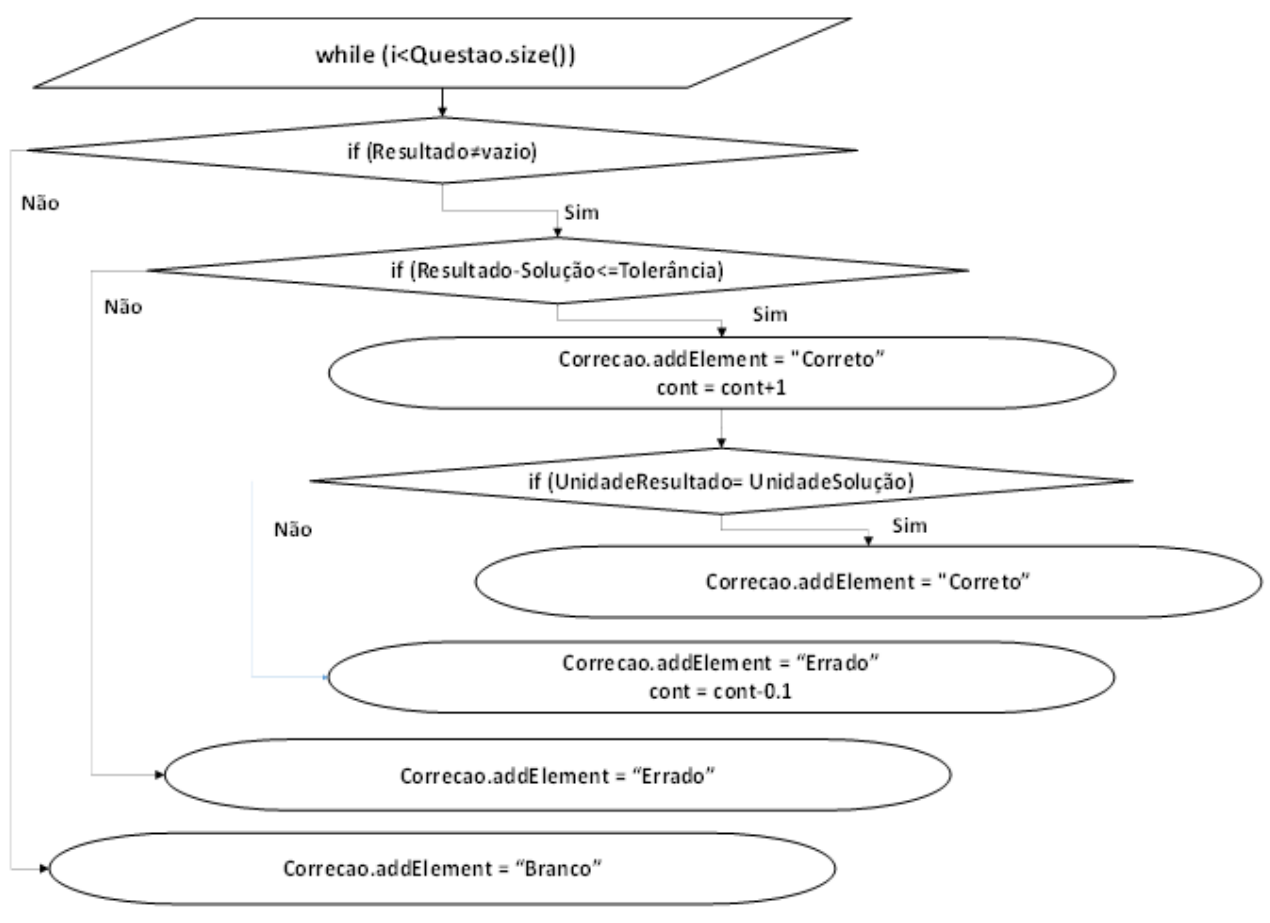

Fonte: autoria própria

A Figura 6 mostra que a implementação da lógica de correção conseguiu realizar a comparação das respostas numéricas e unidades com a solução do banco de questões considerando a tolerância de cada item. Definiu também os status dos itens como correto, errado ou branco e contabilizou a nota percentual da questão.

Foi proposto que no momento que o aluno clica em gerar a questão, o software cria um arquivo que guarda o número aleatório da questão, garantindo o back-up da questão em caso de panes.

Finalmente foi aplicado um questionário aos discentes solicitando a opinião sobre o sistema de avaliação da disciplina, na Tabela 1 tem-se as respostas do questionário envolvendo o método utilizado com uso do software.

A Tabela 1 mostra que os discentes são favoráveis a metodologia de avaliação que emprega os ciclos de aprendizado, avaliação e diagnóstico, com 100\% dos resultados favoráveis.

Tabela 1 - Quantidade percentual das respostas do questionário pertinentes ao software

\begin{tabular}{l|c|c|c|c|c|c}
\hline Pergunta & Ótimo & Boa & Regular & Ruim & Péssima & Outra \\
\hline $\begin{array}{l}\text { Qual é a sua opinião } \\
\text { sobre o sistema de } \\
\text { avaliações utilizadas } \\
\text { na disciplina? }\end{array}$ & $71 \%$ & $29 \%$ & $0 \%$ & $0 \%$ & $0 \%$ & $0 \%$ \\
\hline $\begin{array}{l}\text { Qual é a sua opinião } \\
\text { sobre as provas } \\
\text { digitais aplicadas? }\end{array}$ & $29 \%$ & $14 \%$ & $21 \%$ & $0 \%$ & $7 \%$ & $29 \%$ \\
\hline
\end{tabular}

Fonte: autoria própria 
Uma porcentagem significativa dos alunos também avaliou como ótimo ou bom o emprego das provas digitais utilizando o software, apesar de alguns resultados regulares e péssimos que devem ser considerados nos próximos desenvolvimentos. A resposta "Outra” está associada aos alunos que não realizaram nenhuma prova digital, pois a primeira avaliação era realizada em sala de aula com prova no papel.

\section{RESULTADOS}

O desenvolvimento do software para realizar a criação, a correção e o diagnóstico das avaliações com uma interface amigável foi possível graças a versatilidade da linguagem Java, permitindo apresentar de forma clara e organizada tanto os enunciados e as figuras explicativas quanto os itens com os campos de respostas dos alunos. Outro ponto relevante está associado a aba de resultados que apresenta de forma sintética e organizada os resultados alcançados pelos alunos e o status da correção, além da nota percentual das questões da avaliação como um todo. Também foi possível a criação de um relatório em pdf para documentar a avaliação de maneira semelhante as provas físicas.

Os discentes da disciplina também foram favoráveis a aplicação da metodologia de avaliação em ciclos de aprendizados e avaliaram de maneira satisfatória a aplicação do software de avaliação digital.

Em termos da melhoria do aprendizado para evitar retenção, o software permitiu a correção dinâmica das avaliações e a apresentação do retorno imediato do resultado do aprendizado. Assim no final de cada avalição o docente realizava a análise das questões focadas no aprendizado do conteúdo, sendo que a grande maioria da recuperação dos alunos ocorreu na segunda avaliação, o que será exposto em outro trabalho.

Outro ponto relevante na digitalização das avaliações é a redução dos custos e do impacto ambiental de impressão e de reprodução das provas, além de apresentar um impacto social favorável com emprego destes recursos em outras atividades da Universidade.

\section{REFERÊNCIAS}

ABOUTBOUl, H. O Sistema de Avaliação como Componente do Processo de Formação do Engenheiro. Revista de Ensino em Engenharia, São Paulo 4(1):82-84,1985.

BURINI, E. R. V.; PINHEIRO, A. C. F. B. Avaliação como Medida do Aprendizado no

Ensino da Engenharia. XXX Congresso Cobenge da ABENGE, Rio de Janeiro, 2003.

LIMA, T. L. Avaliação da aprendizagem no ensino superior: contrapontos entre teorias, legislações e exames oficiais. Diálogos \& Ciência (Online), v. 1, p. 86-103, 2016.

LUCKESI, C. C. O que é mesmo o Ato de Avaliar a Aprendizagem. Pátio. Porto alegre: ARTMED. Ano 3, n. 12 fev./abr. 2000.

LUCKESI, C. C. Verificação ou Avaliação:O Que Pratica a Escola? V Congresso Internacional em Avaliação Educacional, 2010, <disponível em:

http://titan2.ccv.ufc.br/newpage/conc/seduc2010/seduc_dir/download/ avaliacao1.pdf > 
LUCKESI, C. C. Avaliação da aprendizagem escolar: Estudos e Proposições. 12aed. São Paulo, Cortez 2011.

ORACLE. O que é a tecnologia Java e porque preciso dela? Disponível em: https://www.java.com/pt_BR/download/faq/whatis_java.xml. Acesso em: 07 jul. 2020.

PIMENTA, S.G.; ANASTASIOU, L.G.C.; CAVALLET, V.J. Docência no ensino superior: construindo caminhos. Disponível em:

https://www.researchgate.net/publication/333614428_Docencia_no_ensino_superior_construindo_ caminhos. Acesso em: 05/07/2020.

SIERRA, K.; BATES, B, Use a Cabeça! Java, Tradução da segunda Edição. Editora Alta Books, 2005. TECNOLOGIA E INFORMAÇÃO, Como o Java Funciona? 2019. Disponível em: $<$ https://tecnologiaeinformacao.netlify.com/_pages/java-b/funcionamento.html>. Acesso em: 08/04/2020.

\title{
IMPLEMENTATION OF A DIGITAL EVALUATION METHOD IN PHYSICAL PHENOMENA DISCIPLINES
}

\begin{abstract}
The teaching and learning process necessarily involves the use of a flexible and efficient assessment method. This method should make it possible to diagnose learning and direct students with unsatisfactory performance to new cycles and learning, assessment and diagnosis. The disciplines of analysis of transport phenomena and unitary operations, present large classes with high retention and evasion rates. In this work, an evaluation method was elaborated and implemented, with the use of software with specific purpose, for the creation and dynamic correction of activities, making possible the various cycles of learning, evaluation and diagnosis for large classes. The software used enabled the dynamic creation of evaluations based on a drawing of questions from a database, performing automatic correction and presenting the results in a diagnostic interface, allowing, at the end of the evaluation, to build learning strategies to direct the students. student studies. The results obtained show that the method used, through the friendly interface of the software and with the organized presentation of the questions and the results of the evaluation, was well accepted by the students.
\end{abstract}

Keywords: learning cycles, assessment and diagnosis, dropout and school retention. 